\title{
NEVUS GENITAL ATÍPICO
}

\author{
Patricia DeCastro Lotero MD*
}

\section{Resumen}

Paciente de 22 años que consulta por presentar una pápula pigmentada en el labio mayor izquierdo. La patología muestra un nevus celular genital atípico con la presencia en la unión dermoepidérmica de una proliferación de melanocitos atípicos y tecas con pérdida de cohesión celular. Se discuten los criterios diagnósticos, los parámetros histopatológicos y el diagnóstico diferencial.

Palabras clave: nevus en sitios especiales, nevus genital, vulva, melanoma.

\section{ATYPIC GENITAL NEVUS}

\section{Abstract}

This case corresponds to a 22 year old patient presenting with a pigmented lesion in the labia majora. The histopathology exam showed an atypical genital cellular nevus and melanocyte atypia proliferation and loss of flattened cell cohesion in the dermal-epidermal junction. Diagnostic criteria, histological parameters and differential diagnoses are discussed.

Key words: nevi of special sites, genital nevus, vulva, melanoma.

\section{CASO CLÍNICO}

Una mujer de 22 años de edad acude a ginecología para su revisión anual. Durante el examen se observa la presencia de una lesión névica vulvar y es remitida a la consulta dermatológica para su valoración. La paciente no lo había detectado por lo cual no se conoce el tiempo de evolución.

Fecha recibido: julio 25 de 2013 - Fecha aceptado: agosto 13 de 2013

* Dermatóloga Dermatopatóloga en proceso de homologación, Universidad CES, Medellín, Colombia. Docente de Dermatopatología de la Fundación Universitaria de Ciencias de la Salud. Bogotá, DC. Colombia.
En la consulta dermatológica al examen físico se identifica en el labio mayor izquierdo una pápula de $6 \mathrm{~mm}$ de diámetro, simétrica, de pigmentación homogénea y bordes levemente irregulares (Figura 1). Se realiza biopsia de la lesión.

Al estudio microscópico los cortes de piel muestran una lesión simétrica y circunscrita con compromiso epidérmico y dérmico. A nivel de la unión dermoepidérmica hay una proliferación de melanocitos aislados y agrupados en tecas, las cuales son de diferentes tamaños, algunas confluyen, con 
pigmento melánico en su interior y pérdida de cohesión celular (Figura 2). La proliferación de melanocitos aislados presenta núcleos grandes hipercromáticos (Figura 3) y hay leve migración pagetoide. A nivel de la dermis las tecas de células névicas presentan abundante pigmento melánico en su interior, hay células névicas multinucleadas en la dermis papilar (Figura 4) y maduración hacia la profundidad. Se hace un diagnóstico de nevus celular genital atípico y se recomienda la extirpación completa de la lesión.

\section{NEVUS CELULAR GENITAL ATÍPICO}

Pertenece al grupo de nevus en sitios especiales. Se presentan en sitios anatómicos definidos y tienen hallazgos histológicos atípicos que llevan a un diagnóstico diferencial con un melanoma o un nevus displásico. Los sitios anatómicos frecuentes son región genital (vulva y pene), pliegues de flexión (inguinal, antecubital y retropoplíteo), la línea mamaria (axila y senos) y las zonas acrales (palmas y plantas). También se han descrito en cuero cabelludo, ombligo, orejas, codos y rodillas. ${ }^{1}$ No todos los que aparecen en estos sitios anatómicos tienen las características histológicas de atipia. La identificación de estos parámetros histológicos es importante para diferenciarlo del melanoma, pues los nevus en estos sitios tienen un comportamiento benigno. ${ }^{2}$

Los nevus melanocíticos vulvares son raros y representan solo el $2.3 \%$ de lesiones pigmentadas ${ }^{3}$ que en la zona genital incluyen léntigos, nevus melanocíticos comunes y displásicos, nevus de Spitz y melanoma. ${ }^{4}$ El nevus genital atípico por lo regular es detectado durante chequeos dermatológicos en pacientes con antecedentes familiares de melanoma o personales de nevus displásico o en chequeos ginecológicos de rutina. Fue descrito en 1981 por Friedman y Ackerman cuando vieron una diferencia histológica entre los nevus en pre y postmenopáusicas. ${ }^{5}$

Su presentación es en jóvenes premenopaúsicas, más del $50 \%$ se ven en menores de 20 años. La vulva (labios mayores, labios menores y clítoris) son los sitios

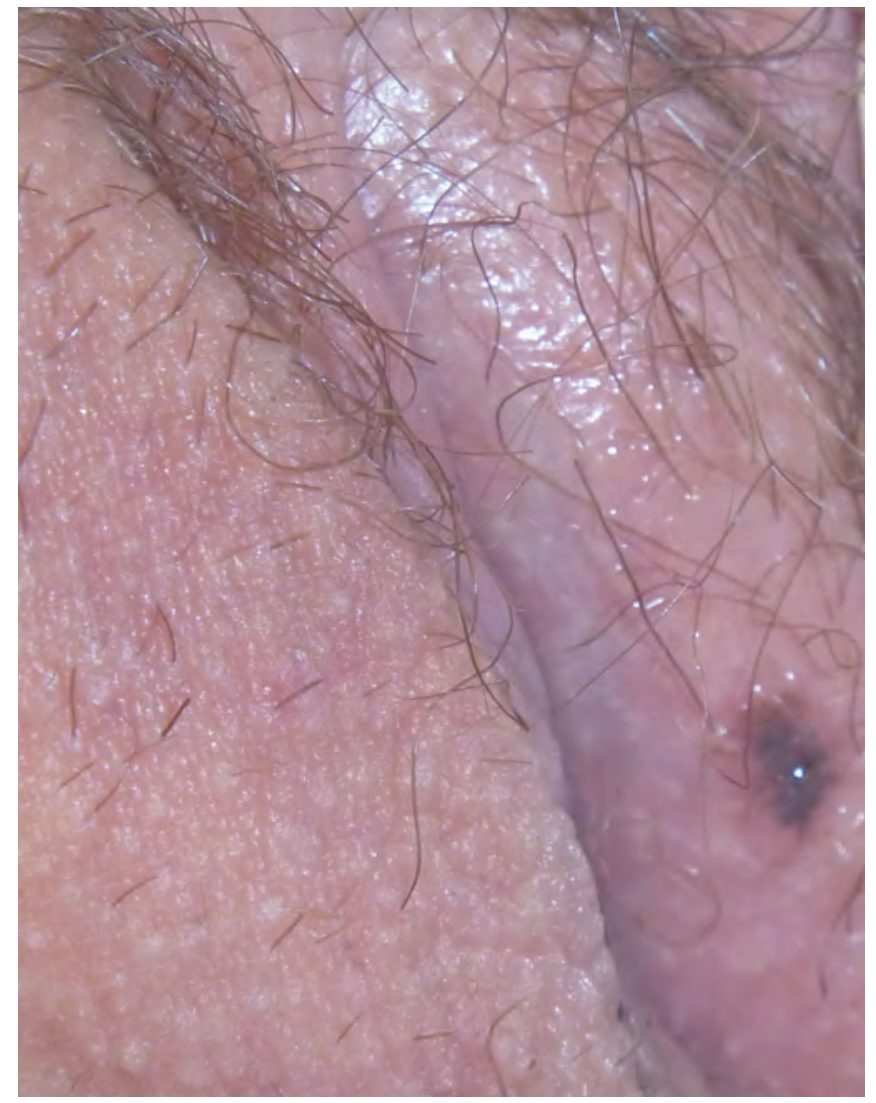

Figura I. Foto clínica: pápula hiperpigmentada en labio mayor izquierdo.

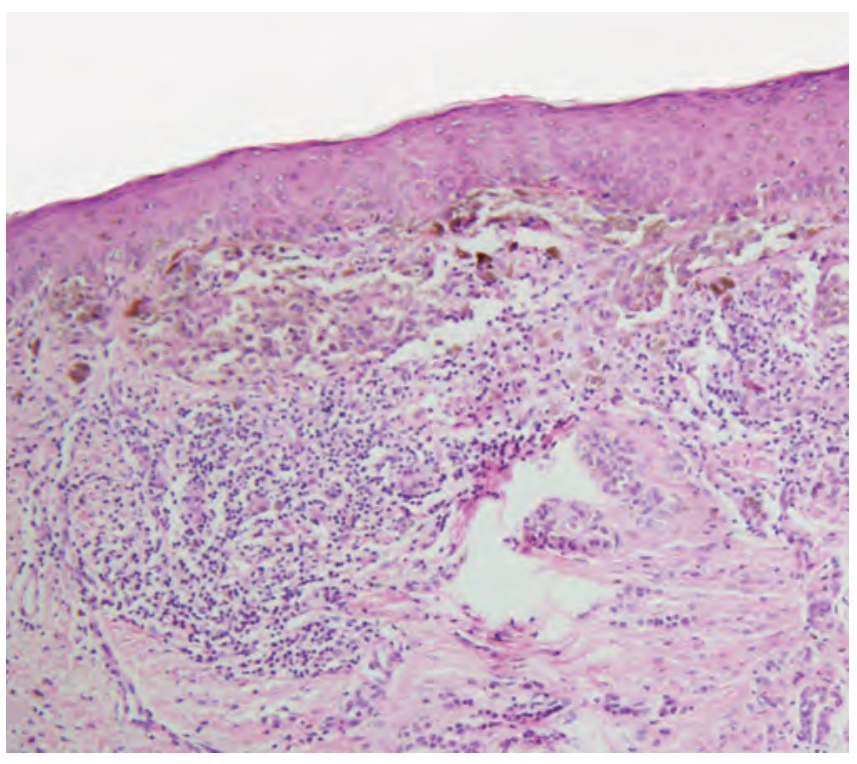

Figura 2. En la unión dermoepidérmica se observan tecas de células névicas con pérdida de la cohesión celular. 


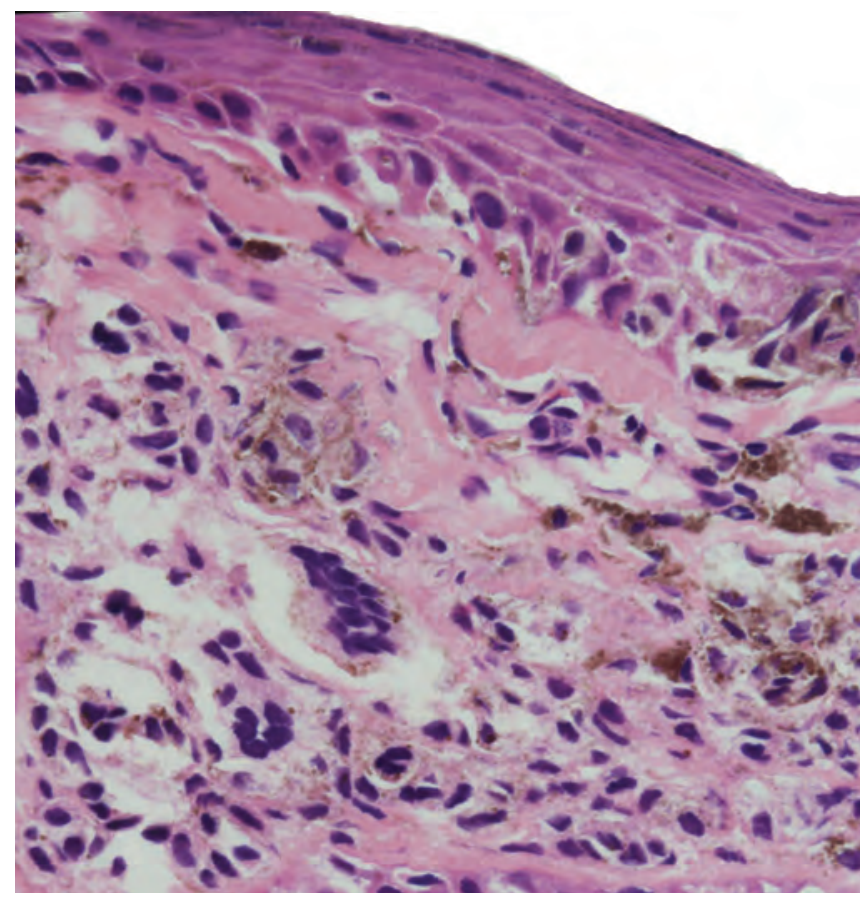

Figura 3.Melanocíticos de núcleos grandes e hipercromáticos en la unión dermoepidérmica.

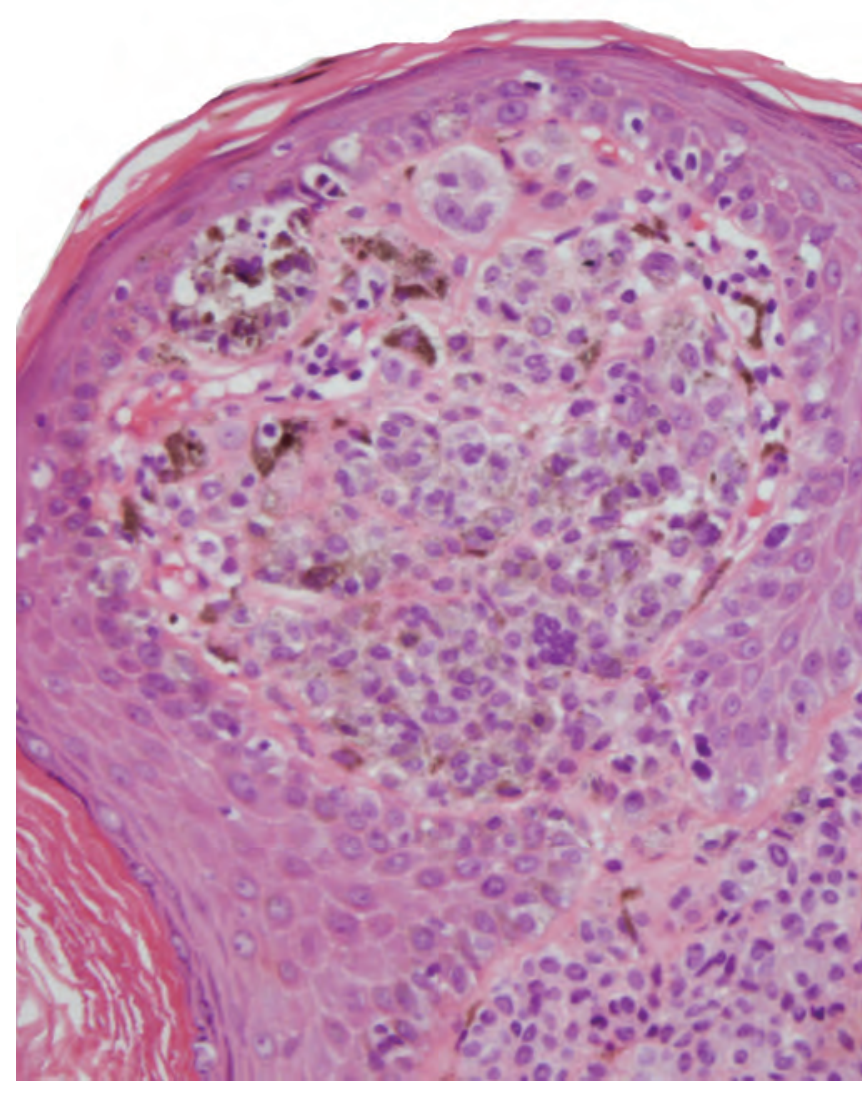

Figura 4. Hiperplasia melanocítica en la unión dermoepidérmica. Presencia de células multinucleadas en la dermis papilar. de presentación más frecuentes, seguido de la región púbica y el periné. ${ }^{3}$

La presentación clínica es el de un nevus melanocítico común, asintomático y puede ser una mácula, pápula o lesión polipoide con pigmentación uniforme y bordes bien delimitados, con tamaño menor de 1 $\mathrm{cm}$. En un estudio donde se revisaron 56 nevus del tracto genital femenino se encontró que el tamaño variaba entre 2 y $20 \mathrm{~mm}$ con un promedio de $6 \mathrm{~mm} .^{6}$

Los hallazgos histológicos atípicos representan una curiosidad histológica en una minoría de nevus genitales. Desde el punto de vista histológico se caracteriza por ser compuesto, bien circunscrito y simétrico. Son lesiones nodulares que al bajo poder llama la atención el aumento en la celularidad y aumento en la pigmentación. ${ }^{3}$ Tienen un componente de unión prominente donde se observan grandes tecas de células névicas de tamaño y forma variable con retracción del tejido vecino y pérdida de cohesión celular. ${ }^{6}$ Los nidos están irregularmente distribuidos en la unión dermoepidérmica y pueden fusionarse formando puentes. Toman aspecto fusiforme $u$ oval con orientación paralela a la membrana basal. El crecimiento lentiginoso de la epidermis y la migración pagetoide hasta la capa granular son hallazgos focales y limitados al centro de la lesión. ${ }^{3}$ Hay compromiso del epitelio folicular en forma de células aisladas o en tecas. Se observa maduración celular hacia la profundidad.

A nivel de la unión dermoepidérmica hay atipia de los melanocitos aislados que va de leve a severa. Son melanocitos epitelioides con citoplasma abundante y eosinofílico y nucléolos prominentes. Se pueden ver melanocitos poligonales de poco citoplasma con núcleos hipercromáticos y presencia de formas multinucleadas. ${ }^{3}$ En la revisión de los 56 casos de nevus genitales atípicos se encontró que el $46 \%$ tenía compromiso de estructuras anexiales, $25 \%$ con células multinucleadas, $18 \%$ migración pagetoide y $7 \%$ mitosis. $^{6}$

El componente dérmico está bien circunscrito con bordes laterales bien delimitados. ${ }^{7}$ La actividad mitótica es baja con menos de dos mitosis por sección 
tisular. No se identifican mitosis en la dermis reticular ni formas atípicas y hay maduración citológica en profundidad. El infiltrado linfocítico puede ser leve o denso. Un hallazgo que preocupa es la presencia de melanocitos en el estroma fibroso en la base de la lesión. $^{7}$

Algunos de los hallazgos histológicos que se identifican en los nevus displásicos pueden estar presentes en el genital atípico. Esto se observó en el estudio que hizo Gleason y col. ${ }^{6}$ donde encontraron que en $61 \%$ de estos hay puentes entre las crestas interpapilares, el $41 \%$ con un hombro de extensión del componente epidérmico más allá del dérmico, el $50 \%$ presentó una proliferación de melanocitos aislados en la unión dermoepidérmica y el $45 \%$ fibrosis dérmica.

Wallace Clark y col. ${ }^{8}$ describieron tres patrones diferentes en este tipo de nevus genital atípico: en nidos, en nidos con pérdida de la cohesión celular y el congestivo. El más frecuente es el primero caracterizado por la presencia de nidos de diferentes tamaños, de orientación perpendicular y paralela a la unión dermoepidérmica. El segundo en frecuencia es el de nidos con pérdida de cohesión celular donde se observan células névicas separadas entre sí en ocasiones formando una banda. Y el patrón congestivo se caracteriza por la mezcla densa de tecas y melanocitos aislados dispuestos en la unión dermoepidérmica. Lo frecuente es ver una mezcla de los tres patrones.

Para hacer un diagnóstico diferencial con el melanoma hay que tener en cuenta parámetros arquitecturales y citológicos. Los primeros en el nevus genital atípico son tamaño pequeño, ausencia de hombro epidérmico y de componente lentiginoso en la epidermis, poca migración pagetoide, componente simetrico en la unión dermoepidermica y en la dermis. En cuanto a los criterios citológicos se debe tener en cuenta que en el nevus celular genital atípico las células névicas atípicas son siempre superficiales, hay maduración citológica hacia la profundidad, pocas mitosis y si están presentes siempre son superficiales, y ausencia de necrosis. ${ }^{9}$

El comportamiento de este nevus genital atípico es de benignidad y no se han reportado casos de melanoma posterior. ${ }^{4}$ La importancia de identificar este nevus es evitar errores diagnósticos llevando a cirugías extensas innecesarias.

\section{Referencias}

1. Mason A, Mohr M, Koch L, Hood A. Nevi of special sites. Clin Lab Med. 2011 Jun;31(2):229-42.

2. Hosler G, Moresi J, Barrett T. Nevi with site related atypia:a review of melanocytic nevi with atypical histologic features based on anatomic site. J Cutan Pathol. 2008; 35(10):889-98.

3. Brenn T. Atypical genital nevus. Arch Pathol Lab Med. 2011 Mar;135 (3):317-20.

4. Ribé A. Melanocytic lesions of the genital área with attention given to atypical genital nevi. J Cutan Pathol. 2008; suppl.2: 24-7.

5. Friedman RJ, Ackerman AB. Difficulties in the histologic diagnosis of melanocytic nevi on the vulvae of premenopausal women. In: Ackerman AB, editor. Pathology of Malignant Melanoma. New York, NY: Masson; 1981. p. 119.

6. Gleason BC, Hirsch MS, Nucci MR, Schmidt BA, Zembowicz A, Mihm MC Jr, t al. Atypical genital Nevi: A Clinicopathologic Analysis of 56 cases. Am J Surg Pathol .2008; 32(1): 51-7.

7. Massi G, LeBoit PE. Nevi on genital skin. In: Histological diagnosis of nevi and Melanoma. Germany: Springer; 2004: 303-14

8. Clark WH Jr, Hood AF, Tucker MA, Jampel RM. Atypical melanocytic nevi of the genital type with a discussion of reciprocal parenchymal stromal interactions in the biology of neoplasia. Hum Pathol. 1998; 29(1)(suppl 1):S1-S24

9. Elder SD. Precursors to melanoma and their mimics: nevi of special sites. Mod Pathol. 2006; 19 (suppl 2):S4-S20. 